\title{
Periodic solutions of singular nonautonomous second order differential equations
}

\author{
Abdelkader BOUCHERIF \\ Differential Equations Research Lab. \\ Department of Mathematical Sciences \\ King Fahd University of Petroleum and Minerals \\ Box: 5046 \\ Dhahran 31261, Saudi Arabia \\ e-mail: aboucher@kfupm.edu.sa
Naima DAOUDI-MERZAGUI
University of Tlemcen \\ Department of Mathematics \\ Box: 119 \\ Tlemcen 13000, Algeria
}

\begin{abstract}
We consider a class of singular nonautonomous second order differential equations. We prove the existence of at least one periodic solution. We use a variational approach based on the mountain pass theorem.

2000 Mathematics Subject Classification: 34B15 - 34C15 - 34C25 - 58E05. Key words: Periodic solutions, nonautonomous differential equations, singular nonlinearities, mountain pass theory.
\end{abstract}

\section{Introduction}

This paper is devoted to the study of the existence of solutions of the following periodic boundary value problem

$$
\left\{\begin{array}{c}
u^{\prime \prime}(t)+f(t, u(t))=g(t) \quad 0<t<2 \pi \\
u(0)-u(2 \pi)=u^{\prime}(0)-u^{\prime}(2 \pi)=0
\end{array}\right.
$$


where $g$ is a bounded measurable function, and $f:[0,2 \pi] \times\left(u_{0},+\infty\right) \rightarrow \mathbb{R}$ is continuous, $2 \pi$-periodic in $t$ and $f(t,$.$) is singular at u_{0} \in \mathbb{R}$; the singularity consists in the fact that the nonlinearity $f(t,$.$) is unbounded from below in a$ neighborhood of $u_{0}$.

Nonlinear second order differential equations with singular restoring forces describe, among other things, the dynamics of particules under the action of Newtonian type forces caused by compressed gazes. Singular two-point boundary value problems have received a great deal of attention. For a good account on recent works we refer the reader to the monographs [1], [15] and the memoire [2].

The problem of existence of periodic solutions of autonomous and nonautonomous singular second order differential equations, with or without a friction term, have been investigated by many authors using a topological method based on the topological degree theory and upper-lower solutions method. See for instance $[3-10],[12],[14],[17],[21]$ and [22]. In almost all these papers the differential equation contains a friction term that does not allow the application of a variational method. Problem (1.1) is treated as a particular case. See for example [3] and [12]. However, our assumptions are different, and moreover, the novelty in our study consists in the application of a variational approach based on the mountain pass theorem. The advantage, here, is that we are able to consider the case when the singular nonlinearity $f(t,$.$) is bounded from above that does$ not meet the conditions to apply the topological approach, see for instance the assumption (H4) in [4] and the assumptions of Theorem 4.1 in [3]. More precisely, we state sufficient conditions on $f$ such that solutions of (1.1) will be sought as critical points of some functional in an appropriate Sobolev space. Moreover, this functional is shown to have a mountain pass geometry. In fact, we shall use a variant of the mountain pass theorem as stated in [18]. For definitions and results on critical point theory, we refer to [11], [13], [18]. Other variants of the mountain pass theorem can be found in [16], [19] and [20].

Finally, we should point out that our main result cannot be deduced trivially from all the above cited works. It complementes quite well the results in [3], [4] and $[12]$.

\section{Preliminaries}

Let $F(t, u):=\int_{1+u_{0}}^{u} f(t, s) d s$. Then problem (1.1) has a variational structure with corresponding functional $\varphi$ given by

$$
\varphi(u):=\int_{0}^{2 \pi}\left[\frac{1}{2} u^{\prime}(t)^{2}-F(t, u(t))+g(t) u(t)\right] d t
$$

and is defined on the Banach space (in fact it is a Hilbert space)

$H_{2 \pi}^{1}:=\left\{u:[0,2 \pi] \rightarrow \mathbb{R}\right.$ absolutely continuous; $\left.u(0)=u(2 \pi), u^{\prime} \in L^{2}([0,2 \pi] ; \mathbb{R})\right\}$, 
equipped with the norm

$$
\|u\|=\left(\int_{0}^{2 \pi}|u(t)|^{2} d t+\int_{0}^{2 \pi}\left|u^{\prime}(t)\right|^{2} d t\right)^{\frac{1}{2}}
$$

for $u \in H_{2 \pi}^{1}$.

It is well known (see for instance [13]) that $\varphi$ is well defined on $H_{2 \pi}^{1}$, continuously differentiable and weakly lower semicontinuous. Moreover, the critical points of $\varphi$ (i.e. $u \in H_{2 \pi}^{1}$ such that $\varphi^{\prime}(u)=0$ ) are solutions of (1.1).

In our work, we shall use a variant of the mountain pass theorem (see [18] for details) to prove our main result.

\section{Main Result}

Consider problem (1.1) with $g:[0,2 \pi] \rightarrow \mathbb{R}$ is a bounded measurable function, and $f:[0,2 \pi] \times\left(u_{0},+\infty\right) \longrightarrow \mathbb{R}$ is continuous and satisfies

(H1) $\lim _{u \rightarrow u_{0}^{+}} f(t, u)=-\infty$ (uniformly in $t$ )

(H2) $\lim _{u \rightarrow u_{0}^{+}} F(t, u)=+\infty$ (uniformly in $t$ )

(H3) $M(t):=\sup \left\{f(t, s) ; u_{0}<s<+\infty\right\}$ is bounded

(H4) $\lim _{u \rightarrow+\infty} \int_{0}^{2 \pi}[F(t, u)-g(t) u] d t=+\infty$

(H5) $D_{1} F(t, u):=\frac{\partial F}{\partial t}(t, u)$ exists and is nonnegative

The above problem was considered in [4] in the case $f$ unbounded above and satisfying $(\mathrm{H} 1),(\mathrm{H} 2),(\mathrm{H} 5)$ and $\lim _{u \rightarrow+\infty} 2 \frac{F(t, u)}{u^{2}}=\mu(t), \mu(t)>0$ such that $\mu_{0}=\sup _{t} \mu(t)<\frac{1}{4}$, using a topological approach based on the topological degree theory and upper-lower solutions method. The fact that the nonlinearity is unbounded from above and from below allows the author in [4] to exhibit a constant upper solution and a constant lower solution, and then construct a set $\Omega$, which is admissible for the use of the topological degree. In the present paper, we consider problem (1.1) when $f$ is bounded above, i.e. we suppose that $M(t):=\sup \left\{f(t, s) ; u_{0}<s<+\infty\right\} \leq M<+\infty$. In this case we cannot use the approach of [4].

Our main result reads as follows.

Theorem A. Assume (H1), (H2), (H3), (H4) and (H5) are satisfied. Then problem (1.1) has at least one solution. 
Proof. The proof will be based on several claims.

For $\lambda \in\left(u_{0}, u_{0}+1\right)$ we consider the following modified problem

$$
\left\{\begin{array}{c}
u^{\prime \prime}(t)+f_{\lambda}(t, u(t))=g(t) \quad 0<t<2 \pi \\
u(0)-u(2 \pi)=u^{\prime}(0)-u^{\prime}(2 \pi)=0
\end{array}\right.
$$

where $f_{\lambda}:[0,2 \pi] \times \mathbb{R} \longrightarrow \mathbb{R}$ is defined by

$$
f_{\lambda}(t, u)= \begin{cases}f(t, u) & u \geq \lambda \\ f(t, \lambda) & u<\lambda\end{cases}
$$

Let $F_{\lambda}(t, u)=\int_{u_{0}+1}^{u} f_{\lambda}(t, s) d s$ and consider the functional

$$
\varphi_{\lambda}: H_{2 \pi}^{1} \longrightarrow \mathbb{R}
$$

defined by

$$
\varphi_{\lambda}(u)=\int_{0}^{2 \pi}\left[\frac{1}{2} u^{\prime}(t)^{2}-F_{\lambda}(t, u(t))+g(t) u(t)\right] d t .
$$

It is well known (see for instance [13]) that $\varphi_{\lambda}$ is well defined on $H_{2 \pi}^{1}$, continuously differentiable and weakly lower semicontinuous. Moreover, the critical points of $\varphi_{\lambda}$ are solutions of (3.1).

Claim 1. $\varphi_{\lambda}$ satisfies the Palais-Smale condition.

Let $\left\{u_{n}\right\}_{n \in \mathbb{N}}$ be a sequence in $H_{2 \pi}^{1}$ such that $\left\{\varphi_{\lambda}\left(u_{n}\right)\right\}_{n \in \mathbb{N}}$ is bounded and $\varphi_{\lambda}^{\prime}\left(u_{n}\right) \longrightarrow 0$ as $n \rightarrow+\infty$ weakly in $H_{2 \pi}^{1}$; i.e. there exist a constant $c_{1}>0$ and a sequence $\left\{\epsilon_{n}\right\}_{n \in \mathbb{N}} \subset \mathbb{R}_{+}$with $\epsilon_{n} \rightarrow 0$ as $n \rightarrow+\infty$ such that

$$
\left|\int_{0}^{2 \pi}\left[\frac{1}{2} u_{n}^{\prime}(t)^{2}-F_{\lambda}\left(t, u_{n}(t)\right)+g(t) u_{n}(t)\right] d t\right| \leq c_{1} \text { for all } n,
$$

and for every $v \epsilon H_{2 \pi}^{1}$,

$$
\left|\int_{0}^{2 \pi}\left[u_{n}^{\prime}(t) v^{\prime}(t)-f_{\lambda}\left(t, u_{n}(t)\right) v(t)+g(t) v(t)\right] d t\right| \leq \epsilon_{n}\|v\|_{H_{2 \pi}^{1}} .
$$

We show that $\left\{u_{n}\right\}_{n \in \mathbb{N}}$ has a bounded subsequence in $H_{2 \pi}^{1}$, and this will be enough to derive the Palais-Smale condition.

Taking $v(t) \equiv-1$ in (3.4) we obtain

$$
\left|\int_{0}^{2 \pi}\left[f_{\lambda}\left(t, u_{n}(t)\right)-g(t)\right] d t\right| \leq \epsilon_{n} \sqrt{2 \pi} \text { for all } n \text {. }
$$


So that

$$
\left|\int_{0}^{2 \pi} f_{\lambda}\left(t, u_{n}(t)\right) d t\right| \leq \epsilon_{n} \sqrt{2 \pi}+\left|\int_{0}^{2 \pi} g(t) d t\right|:=c_{2} .
$$

Let

$$
I_{1, n}:=\left\{t \in[0,2 \pi] ; \quad f_{\lambda}\left(t, u_{n}(t)\right) \geq 0\right\}
$$

and

$$
I_{2, n}:=\left\{t \in[0,2 \pi] ; \quad f_{\lambda}\left(t, u_{n}(t)\right)<0\right\} .
$$

It follows from (3.5) that

$$
\left|\int_{I_{2, n}} f_{\lambda}\left(t, u_{n}(t)\right) d t\right| \leq c_{2}+\int_{I_{1, n}} f_{\lambda}\left(t, u_{n}(t)\right) d t \leq c_{2}+2 \pi M,
$$

where $M$ is such that $M(t) \leq M$ for all $t \in[0,2 \pi]$.

Hence, there exists $c_{3}>0$ such that

$$
\int_{0}^{2 \pi}\left|f_{\lambda}\left(t, u_{n}(t)\right)\right| d t \leq c_{3} \text { for all } n .
$$

On the other hand, if we take, in $(3.4), v(t) \equiv w_{n}(t):=u_{n}(t)-\bar{u}_{n}$, where $\bar{u}_{n}$ is the average of $u_{n}$ over the interval $[0,2 \pi]$,we get (taking into account $(3.6)$ )

$$
\begin{aligned}
c_{4}\left\|w_{n}\right\|_{H_{2 \pi}^{1}} & \geq \int_{0}^{2 \pi}\left[\frac{1}{2} w_{n}^{\prime}(t)^{2}-f_{\lambda}\left(t, u_{n}(t)\right) w_{n}(t)+g(t) w_{n}(t)\right] d t \\
& \geq \frac{1}{2}\left\|w_{n}^{\prime}\right\|_{L^{2}}^{2}-\left(c_{3}+\|g\|_{L^{1}}\right)\left\|w_{n}\right\|_{L^{\infty}} \\
& \geq \frac{1}{2}\left\|w_{n}^{\prime}\right\|_{L^{2}}^{2}-c_{5}\left\|w_{n}\right\|_{H_{2 \pi}^{1}} .
\end{aligned}
$$

Consequently, using the Poincaré-Wirtinger inequality for zero mean functions in the Sobolev space $H_{2 \pi}^{1}$, there exists $c_{6}>0$ such that

$$
\left\|u_{n}^{\prime}\right\|_{L^{2}} \leq\left\|w_{n}\right\|_{H_{2 \pi}^{1}} \leq c_{6}
$$

Suppose, now, that

$$
\left\|u_{n}\right\|_{H_{2 \pi}^{1}} \rightarrow+\infty \text { as } n \rightarrow+\infty
$$

Since (3.7) holds, we have, passing to subsequences if necessary, that either

$$
\begin{aligned}
& m_{n}:=\min u_{n} \rightarrow-\infty \text { as } n \rightarrow+\infty, \text { or } \\
& M_{n}:=\max u_{n} \rightarrow+\infty \text { as } n \rightarrow+\infty .
\end{aligned}
$$


(i) Assume that the second possibility occurs. We have

$$
\begin{aligned}
\int_{0}^{2 \pi} & {\left[F_{\lambda}\left(t, u_{n}(t)\right)-g(t) u_{n}(t)\right] d t } \\
& =\int_{0}^{2 \pi}\left[\left(\int_{1+u_{0}}^{u_{n}(t)} f_{\lambda}(t, s) d s\right)-g(t) u_{n}(t)\right] d t \\
& =\int_{0}^{2 \pi}\left[\left(\int_{1+u_{0}}^{M_{n}} f_{\lambda}(t, s) d s-\int_{u_{n}(t)}^{M_{n}} f_{\lambda}(t, s) d s\right)-g(t) u_{n}(t)\right] d t \\
& =\int_{0}^{2 \pi}\left[F_{\lambda}\left(t, M_{n}\right)-M_{n} g(t)\right] d t-\int_{0}^{2 \pi}\left[\int_{u_{n}(t)}^{M_{n}}\left(f_{\lambda}(t, s)-g(t)\right) d s\right] d t \\
& \geq \int_{0}^{2 \pi}\left[F_{\lambda}\left(t, M_{n}\right)-M_{n} g(t)\right] d t-\left\|M_{\lambda}-g\right\|_{L^{1}}\left\|M_{n}-u_{n}\right\|_{C} .
\end{aligned}
$$

$\left(\right.$ Here $\left.M_{\lambda}(t)=\sup \left\{f_{\lambda}(t, s) ; u_{0}<s<+\infty\right\}\right)$.

Thus, applying Sobolev and Poincaré's inequalities to $M_{n}-u_{n}(\cdot)$,

$$
\begin{aligned}
\int_{0}^{2 \pi}\left[F_{\lambda}\left(t, M_{n}\right)-\right. & \left.M_{n} g(t)\right] d t \leq \int_{0}^{2 \pi}\left[F_{\lambda}\left(t, u_{n}(t)\right)-g(t) u_{n}(t)\right] d t \\
& +\left\|M_{\lambda}-g\right\|_{L^{1}} \sqrt{2 \pi} c_{6} \text { for all } n .
\end{aligned}
$$

Using (3.3) and (3.7) we see that the sequence

$$
\int_{0}^{2 \pi}\left[F_{\lambda}\left(t, M_{n}\right)-M_{n} g(t)\right] d t \text { is bounded. }
$$

This contradicts (H4).

(ii) Assume the first possibility occurs; i.e. $m_{n} \rightarrow-\infty$ as $n \rightarrow+\infty$. We replace $M_{n}$ by $-m_{n}$ in the preceeding arguments, and we also arrive at a contradiction.

Therefore $\varphi_{\lambda}$ satisfies the Palais-Smale condition. This completes the proof of the claim.

Let

$$
\Omega:=\left\{u \in H_{2 \pi}^{1} ; \min u>1+u_{0}\right\}
$$

and

$$
\begin{gathered}
\partial \Omega=\left\{u \in H_{2 \pi}^{1} ; u(t) \geq 1+u_{0} \text { for every } t \in(0,2 \pi),\right. \\
\left.\exists t_{u} \in(0,2 \pi): u\left(t_{u}\right)=1+u_{0}\right\} .
\end{gathered}
$$

We now proceed to show that $\varphi_{\lambda}$ has a mountain pass geometry. 
Claim 2. There exists $m>0$ such that $\inf _{u \in \partial \Omega} \varphi_{\lambda}(u) \geq-m$ whenever $\lambda \in\left(u_{0}, u_{0}+1\right)$.

For $u \in \partial \Omega$, we have $\min u=u\left(t_{u}\right)=1+u_{0}$ for some $t_{u}$. Extending the functions by $2 \pi$-periodicity, we can write

$$
\begin{aligned}
\varphi_{\lambda}(u)= & \int_{t_{u}}^{t_{u}+2 \pi}\left[\frac{1}{2} u^{\prime}(t)^{2}-F_{\lambda}(t, u(t))+g(t) u(t)\right] d t \\
\geq & \int_{t_{u}}^{t_{u}+2 \pi} \frac{1}{2} u^{\prime}(t)^{2} d t-\left[\int_{t_{u}}^{t_{u}+2 \pi}\left(M_{\lambda}(t)-g(t)\right)\left(u(t)-u_{0}-1\right) d t\right. \\
& \left.-\int_{t_{u}}^{t_{u}+2 \pi} g(t)\left(u_{0}+1\right) d t\right] .
\end{aligned}
$$

Schwarz inequality and the fact that $u^{\prime}(t)=\left(u(\cdot)-u_{0}-1\right)^{\prime}(t)$ imply $\left.\varphi_{\lambda}(u) \geq \frac{1}{2} \| u(\cdot)-u_{0}-1\right)^{\prime}\left\|_{L^{2}}^{2}-\right\| M_{\lambda}-g\left\|_{L^{2}} \cdot\right\| u()-.u_{0}-1\left\|_{L^{2}}+\left(1+u_{0}\right)\right\| g \|_{L^{1}}$.

Applying Poincaré's inequality to $u(\cdot)-u_{0}-1$, we get

$$
\varphi_{\lambda}(u) \geq \frac{1}{2}\left\|u^{\prime}\right\|_{L^{2}}^{2}-\gamma\left\|M_{\lambda}-g\right\|_{L^{2}}\left\|u^{\prime}\right\|_{L^{2}}+\left(1+u_{0}\right)\|g\|_{L^{1}},
$$

where $\gamma=\gamma\left(t_{u}\right)$.

The above inequality shows that

$$
\varphi_{\lambda}(u) \rightarrow+\infty \text { as }\left\|u^{\prime}\right\|_{L^{2}} \rightarrow+\infty \text {. }
$$

When $\min u=1+u_{0}$, we have that $\left\|u(\cdot)-u_{0}-1\right\|_{H_{2 \pi}^{1}} \rightarrow+\infty$ is equivalent to $\left\|u^{\prime}\right\|_{L^{2}} \rightarrow+\infty$.

- Hence $\varphi_{\lambda}(u) \rightarrow+\infty$ as $\|u\|_{H_{2 \pi}^{1}} \rightarrow+\infty, u \in \partial \Omega$. We infer that $\varphi_{\lambda}$ is coercive, and so it has a minimizing sequence. The weak lower semicontinuity of $\varphi_{\lambda}$ yields

$$
\inf _{u \in \partial \Omega} \varphi_{\lambda}(u)>-\infty .
$$

It follows that there exists $m>0$ such that $\inf _{u \in \partial \Omega} \varphi_{\lambda}(u) \geq-m$, and this is true for all $\lambda \in\left(u_{0}, u_{0}+1\right)$.

The proof of the claim is complete.

Claim 3. There exists $\lambda_{0} \in\left(u_{0}, u_{0}+1\right)$ with the property that for every $\lambda \in\left(u_{0}, \lambda_{0}\right)$, any solution $u$ of $(3.1)$ satisfying $\varphi_{\lambda}(u) \geq-m$ is such that $\min u \geq \lambda_{0}$, and hence $u$ is a solution of (1.1).

For, assume on the contrary that there are sequences $\left\{\lambda_{n}\right\}_{n \in \mathbb{N}}$ and $\left\{u_{n}\right\}_{n \in \mathbb{N}}$ such that 
(i) $\lambda_{n} \leq u_{0}+\frac{1}{n}$

(ii) $u_{n}$ is a solution of (3.1) with $\lambda=\lambda_{n}$

(iii) $\varphi_{\lambda_{n}}\left(u_{n}\right) \geq-m$

(iv) $\min u_{n}<u_{0}+\frac{1}{n}$

Since $f$ is bounded above by $M$ and $\int_{0}^{2 \pi}\left[f_{\lambda_{n}}\left(t, u_{n}(t)\right)-g(t)\right] d t=0$, we have

$$
\left\|f_{\lambda_{n}}\left(\cdot, u_{n}(\cdot)\right)\right\|_{L^{1}} \leq c_{7}, \text { for some constant } c_{7}>0 .
$$

Hence

$$
\left\|u_{n}^{\prime}\right\|_{L^{\infty}} \leq c_{8}, \text { for some constant } c_{8}>0 .
$$

Since $\varphi_{\lambda_{n}}\left(u_{n}\right) \geq-m$ it follows that there must exist two constants $R_{1}$ and $R_{2}$, with $u_{0}<R_{1}<R_{2}$ such that

$$
\max \left\{u_{n}(t) ; t \in[0,2 \pi]\right\} \in\left[R_{1}, R_{2}\right],
$$

otherwise, $u_{n}$ would tend uniformly to $u_{0}$ or $+\infty$, and in this case $\varphi_{\lambda_{n}}\left(u_{n}\right)$ would go to $-\infty$, (because of (H4) and $\left\|u_{n}^{\prime}\right\|_{L^{\infty}} \leq c_{8}$ ), which contradicts $\varphi_{\lambda_{n}}\left(u_{n}\right) \geq-m$.

Let $\tau_{n}^{1}, \tau_{n}^{2}$ be such that, for $n$ large enough

$$
u_{n}\left(\tau_{n}^{1}\right)=u_{0}+\frac{1}{n}<R_{1}=u_{n}\left(\tau_{n}^{2}\right) .
$$

Multiplying the differential equation in (3.1) by $u_{n}^{\prime}$ and integrating the resulting equation on $\left[\tau_{n}^{1}, \tau_{n}^{2}\right]$, or on $\left[\tau_{n}^{2}, \tau_{n}^{1}\right]$, we get

$$
\begin{gathered}
J:=\int_{\tau_{n}^{1}}^{\tau_{n}^{2}} u_{n}^{\prime \prime}(t) u_{n}^{\prime}(t) d t+\int_{\tau_{n}^{1}}^{\tau_{n}^{2}} f_{\lambda_{n}}\left(t, u_{n}(t)\right) u_{n}^{\prime}(t) d t \\
=\int_{\tau_{n}^{1}}^{\tau_{n}^{2}} g(t) u_{n}^{\prime}(t) d t .
\end{gathered}
$$

It is clear that

$$
J=J_{1}+\frac{1}{2}\left[u_{n}^{\prime 2}\left(\tau_{n}^{2}\right)-u_{n}^{\prime 2}\left(\tau_{n}^{1}\right)\right]
$$

where

$$
J_{1}=\int_{\tau_{n}^{1}}^{\tau_{n}^{2}} f_{\lambda_{n}}\left(t, u_{n}(t) u_{n}^{\prime}(t) d t .\right.
$$


Since $g$ is integrable and $\left\|u_{n}^{\prime}\right\|_{L^{\infty}} \leq c_{8}$, it follows that $J$ is bounded, and consequently $J_{1}$ is bounded On the other hand, we have

$$
f_{\lambda_{n}}\left(t, u_{n}(t)\right) u_{n}^{\prime}(t)=\frac{d}{d t}\left[F_{\lambda_{n}}\left(t, u_{n}(t)\right)\right]-D_{1} F_{\lambda_{n}}\left(t, u_{n}(t)\right) .
$$

Thus

$$
J_{1}=F_{\lambda_{n}}\left(\tau_{n}^{2}, R_{1}\right)-F_{\lambda_{n}}\left(\tau_{n}^{1}, u_{0}+\frac{1}{n}\right)-\int_{\tau_{n}^{1}}^{\tau_{n}^{2}} D_{1} F_{\lambda_{n}}\left(t, u_{n}(t)\right) d t .
$$

The assumption (H5) implies that

$$
J_{1} \leq F_{\lambda_{n}}\left(\tau_{n}^{2}, R_{1}\right)-F_{\lambda_{n}}\left(\tau_{n}^{1}, u_{0}+\frac{1}{n}\right) .
$$

It follows from (H2) that $J_{1}$ is not bounded. This is a contradiction.

Claim 4. $\varphi_{\lambda}$ has a mountain-pass geometry for $\lambda \leq \lambda_{0}$.

Fix $\lambda \in\left(u_{0}, \lambda_{0}\right]$ such that $f(t, \lambda)<0$ for any $t \in[0,2 \pi]$. This is possible because of $(\mathrm{H} 1)$.

$$
\begin{aligned}
F_{\lambda}\left(t, u_{0}\right) & =\int_{1+u_{0}}^{u_{0}} f_{\lambda}(t, s) d s=-\int_{u_{0}}^{u_{0}+1} f_{\lambda}(t, s) d s \\
& =-\int_{u_{0}}^{\lambda} f_{\lambda}(t, s) d s-\int_{\lambda}^{u_{0}+1} f_{\lambda}(t, s) d s \\
& =-\int_{u_{0}}^{\lambda} f(t, \lambda) d s-\int_{\lambda}^{u_{0}+1} f_{\lambda}(t, s) d s \\
& =-\left(\lambda-u_{0}\right) f(t, \lambda)-\int_{\lambda}^{u_{0}+1} f_{\lambda}(t, s) d s .
\end{aligned}
$$

This implies that

$$
F_{\lambda}\left(t, u_{0}\right)>-\int_{\lambda}^{u_{0}+1} f(t, s) d s=\int_{u_{0}+1}^{\lambda} f(t, s) d s=F_{\lambda}(t, \lambda) .
$$

Hence

$$
\begin{aligned}
\varphi_{\lambda}\left(u_{0}\right) & =-\int_{0}^{2 \pi} F_{\lambda}\left(t, u_{0}\right) d t+\int_{0}^{2 \pi} g(t) u_{0} d t \\
& <-\int_{0}^{2 \pi} F_{\lambda}(t, \lambda) d t+u_{0}\|g\|_{L^{1}} .
\end{aligned}
$$

Consider $\lambda \in\left(u_{0}, \lambda_{0}\right]$ such that

$$
F_{\lambda}(t, \lambda)>\frac{m+\|g\|_{L^{1}} u_{0}}{2 \pi} \text { for all } t \in[0,2 \pi]
$$

This is possible by (H2). 
It follows that $\varphi_{\lambda}\left(u_{0}\right)<-m-u_{0}\|g\|_{L^{1}}+u_{0}\|g\|_{L^{1}}$ or $\varphi_{\lambda}\left(u_{0}\right)<-m$.

Also, using (H4) we can find $R$, sufficiently large so that $R>1+u_{0}$ and

$$
F_{\lambda}(t, R)>\frac{m+R\|g\|_{L^{1}}}{2 \pi} \text { for all } t \epsilon[0,2 \pi] .
$$

This implies that

$$
\varphi_{\lambda}(R)<-m \text {. }
$$

Since $\Omega$ is a neighborhood of $R, u_{0} \notin \Omega$ and

$$
\max \left\{\varphi_{\lambda}\left(u_{0}\right), \varphi_{\lambda}(R)\right\}<\inf _{u \in \partial \Omega} \varphi_{\lambda}(u)
$$

we are in the situation of the mountain-pass theorem (see [18]).

Claim 1 and Claim 4 imply that $\varphi_{\lambda}$ has a critical point $u_{\lambda}$ such that

$$
\varphi_{\lambda}\left(u_{\lambda}\right)=\inf _{\eta \in \Gamma} \max _{0 \leq s \leq 1} \varphi_{\lambda}(\eta(s)) \geq \inf _{u \in \partial \Omega} \varphi_{\lambda}(u),
$$

where $\Gamma:=\left\{\eta \in C\left([0,1] ; H_{2 \pi}^{1}\right) ; \eta(0)=u_{0}, \eta(1)=R\right\}$.

Since $\inf _{u \in \partial \Omega} \varphi_{\lambda}(u) \geq-m$, it follows from claim 3 that $u_{\lambda}$ is a solution of (1.1).

This completes the proof of the main result.

\section{Acknowledgement}

The authors wish to thank an anonymous referee for suggestions and comments that led to the improvement of the manuscript. Also, A. Boucherif is grateful to KFUPM for its constant support.

\section{References}

[1] R. P. AGARWAL and D. O'REGAN, Singular Differential and Integral Equatons with Applications, Kluwer Acad. Publ., Dordrecht, 2003.

[2] D. BONHEURE, Second Order Periodic Boundary Value Problems with Singularities, Memoire de D.E.A., Louvain-La-Neuve, 2001.

[3] D. BONHEURE and C. DE COSTER, Forced singular oscillatiors and the method of lower and upper solutions, Topological Methods in Nonl. Anal. 22 (2003), 297-317

[4] N. DAOUDI-MERZAGUI, Periodic Solutions of Nonautonomous Second Order Differential Equations with a Singularity, Appl. Anal. 73(3-4) (1999), 449-462. 
[5] M. A. DEL PINO and R. MANASEVICH, Infinitely many T-periodic Solutions for a Problem Arising in Nonlinear Elasticity, J. Diff. Equations 103(2) (1993), 260-277.

[6] R. FAURE, Solutions periodiques d'equations admettant des poles; etude par la methode de Leray-Schauder et par un theoreme de point fixe, C.R. Acad. Sci. Paris 283 A(1975), 481-484.

[7] A. FONDA, Periodic solutions of scalar second order differential equations with a singularity, memoire de la Classe des Sciences, Acad. Royale Belgique, Tome 4 (1993), 5-39.

[8] A. FONDA, R. MANASEVICH and F. ZANOLIN, Subharmonic solutions for some second order differential equations with singularities, S.I.A.M. J. Math. Anal. 24(5) (1993), 1294-1311.

[9] P. HABETS and L. SANCHEZ, Periodic solutions of some Lineard equations with singularities, Proc. Amer. Math. Soc. 109(4) (1990), 1035-1044.

[10] A. C. LAZER and S. SOLIMINI, On periodic solutions of nonlinear differential equations with singulatiries, Proc. Amer. Math. Soc. 99(1) (1987), 109-114.

[11] P. MAJER, Ljusternik-Schnirelmann theory with local Palais-Smale conditions and singular dynamical systems, Ann. Inst. H. Poincare, Anal. Non Lineaire 8 (1991), 459-476.

[12] R. F. MARTINS, Existence of periodic solutions for second order differential equations with singularities and the strong force condition, J. Math. Anal. Appl. 317 (2006), 1-13

[13] J. MAWHIN and M. WILLEM, Critical Point Theory and Hamiltonian Systems, Springer Verlag, New York, 1989.

[14] P. OMARI and W. YE, Necessary and sufficient conditions for the existence of periodic solutions of second order ordinary differential equations with singular nonlinearities, Differential and Integral Eq. 8(7) (1995), 1843-1858.

[15] D. O'REGAN, Theory of Singular Boundary Value Problems, World Sc. Publ. Co., River Edge, New Jersey, 1994.

[16] R. PRECUP, On the Palais-Smale condition for Hammerstein integral equations in Hilbert spaces, Nonl. Anal. 47 (2001), 1233-1244.

[17] R. PRECUP, Periodic solutions of superlinear singular ordinary differential equations, Differential Integral Eq. To appear. 
[18] P. H. RABINOWITZ, Minimax Methods in Critical Point Theory with Applications to Differential Equations, C.B.M.S. Math. 65 Amer. Math. Soc., Providence, R.I., 1986.

[19] M. SCHECHTER, An Introduction to Nonlinear Analysis, Cambridge University Press, 2004

[20] K. TINTAREV, Mountain pass and impasse for nonsmooth functionals with constraints, Nonl. Anal. 27(9) (1996), 1049-1054.

[21] J. Y. WANG and D. Q. JIANG, A singular nonlinear second order periodic boundary value problem, Tohoku Math. J. 50 (1998), 203-210.

[22] M. ZHANG, Periodic solutions of damped differential systems with repulsive singular forces, Proc. Amer. Math. Soc. 127(2) (1999), 401-407.

Received 20 January 2007; accepted 20 January 2007;

published Online First 10 March 2008

To access this journal online:

http://www.birkhauser.ch 